\title{
Model of Carriage Movement on Plane with Dry Friction Forces
}

https://doi.org/10.3991/ijoe.v16i08.14551

G.M. Rosenblat

Moscow Automobile and Road Construction State Technical University (MADI),

Moscow, Russia

V.F. Tishkin

Keldysh Institute of Applied Mathematics, Moscow, Russia

V.B. Yashin $\left({ }^{\bowtie}\right)$,

Moscow Technical University of Communications and Informatics (MTUCI),

Moscow, Russia

hekkoki@gmail.com

\begin{abstract}
Wheel slip model is an important aspect of vehicle driving stability and safety control. However, in most of the carriage movement models friction forces and wheel slip effect are being neglected. This paper raises the problem of wheel slip in dangerous driving and autonomous vehicles under critical driving modes, then tasks the modeling of movement of an individual vehicle is considered. These are two tasks of nonholonomic mechanics: 1) the movement of a wheelset without wheel slip (two disks freely mounted on an axis) along an inclined plane in the field of gravity, and 2) the movement of a flat wheel model, which, under certain assumptions, can be a four-wheeled carriage. In these tasks, in addition to the standard dynamics (continuous motion without wheel slip), critical situations can also be observed associated with wheel sleep and separation of wheels from plane.
\end{abstract}

Keywords-Wheel slip, automobile, traffic accident, dangerous driving, simulation, dry friction, nonholonomic mechanics, carriage model.

\section{$1 \quad$ Introduction}

\subsection{Dangerous driving}

The steady increase in motorization has made personal vehicles widely available. The number of cars in the streets is constantly increasing. Due to the fact that the accident rate of road transport causes enormous physical and financial damage to society in general and citizens in particular, the problem of transport safety is one of the priority tasks for any government [1]. This problem is especially acute in large cities where saturated complex transport networks are overloaded and population density is up to 
maximum limit [2]. There are many reasons why traffic accidents occur. Among them are natural disasters, destruction of the roadway, slippery road surface, failure of traffic lights, ignoring road signs and markings, inattention of road users to the situation on the road and to other road users, state of alcohol or drug affect, strokes and sudden health problems of drivers, as well as an unbalanced mental state of mind or a state of panic. Since the road transport network is a complex system, in order to maintain normal functioning, it requires constant involvement and attention from all its participants [3]. Therefore, any neglect of this complex system in favor of driver's personal interests can cause fatal consequences.

More and more the situation of the constant use of mobile phones while driving is used as an example of such attitude. However, another, no less frequently occurring side of the problem is aggressive driving or driving in a state of panic. Panic and aggressiveness from the psychological point of view lead to the fact when a person should not feel like a member of a group, feel fear for his own life, and begins to act logically, but not in the laws and not according to the rules of groups, but in his own individual manner, violating the coherence of the whole system [4]. In this state, the driver can make decisions that are unpredictable for other participants, so they may either not have time to react, or these actions will provoke emergency or panic actions, which will lead to a dangerous situation or to a collision. This phenomenon has various names: dangerous driving, aggressive driving, careless driving and reckless driving. Laws of many countries provide for various fines and other penalties for such actions. The Government of the Russian Federation identifies the following types of dangerous driving [5]:

- Multiple and sudden lane changes

- Lane change with small gaps (headways)

- Tailgating (decrease of headways)

- Decreasing lateral interval

- Emergency breaking and rubber-necking

- Obstructing overtaking

Nevertheless, despite the apparent clarity of the classification of dangerous driving, there are many questions from the law point of view. As noted on the official website of the Road Traffic Organization of the Russian Federation, "the line between creating a threat is very thin, subjective, and uncertain" [6]. Therefore, in most cases it becomes possible to qualify dangerous driving only after accident occurred. And the punishment for dangerous driving, if it is proved to be the cause of the accident, is additional to the main consequences for the immediate actual accident.

The attempts to formulate the qualitative characteristics of such type of dangerous driving as "Multiple and sudden lane changes" by analyzing the vehicle's trajectory along GPS coordinates were made by our research team in the article [7]. 


\subsection{Autonomous transport}

The high injury risk of road transport caused by the instability of human psychology can be eliminated by increasing the level of autonomy of the car. Most specialists believe that the exclusion of the human factor from the transport system will allow reducing accident rate and optimizing the efficiency of transport networks.

Self-driving cars, or autonomous vehicles (AVs), connected and autonomous vehicles (CAVs), driverless cars, robo-cars or robotic cars, development of which begin history back in the 20s of the last century, have become an obvious trend of our time, and plans for the development and implementation of autonomous vehicles have become part of the national programs of many countries, automotive manufacturing companies, as well as institutes and research centers. Currently, such large projects in this area are known as: Autopilot of Tesla, Cruise of GM, Zoox (California), Nuro (California), Pony.AI (China, Guangzhou), VW, Audi, Symbioz by Renault, BMW, Volvo, Nissan, Waymo by Google, Robo-Taxi by Yandex, Baidu, Almotive (Hungary), AutoX Technologies (Hong Kong and San Jose), WeRide (China, Guangzhou), AKTIV, VisLab, Leonie from Braunschweig, KAMAZ with Cognitive Technologies and others. Also in this regard, one cannot fail to mention such programs for the development of an autonomous vehicle as the European Commission Program, the 2getthere program in the Netherlands, the ARGO program in Italy, and the DARPA Grand Challenge in the USA.

SAE International has established 6 levels of autonomy for self-driving cars (See Fig.1). These levels from zero to fifth characterize the degree of control of artificial intelligence over the vehicle and the degree of human involvement in the process of driving. Level zero is a car without any automation; ABS and ordinary cruise control do not count. Level 1 includes cars with steering and braking control, adaptive cruise control. Level 2 cars can control taxiing and braking in certain areas, such as highways, but the driver must still be vigilant and be ready to take control at any time. Level 3 allows the driver not to watch the headway all the time. Level 4 is already a full autopilot, but it only works in certain conditions and in a marked area, it even allows the driver to take a nap. Level 5 is a complete autopilot without any restrictions. No steering wheel is required at this level [8].

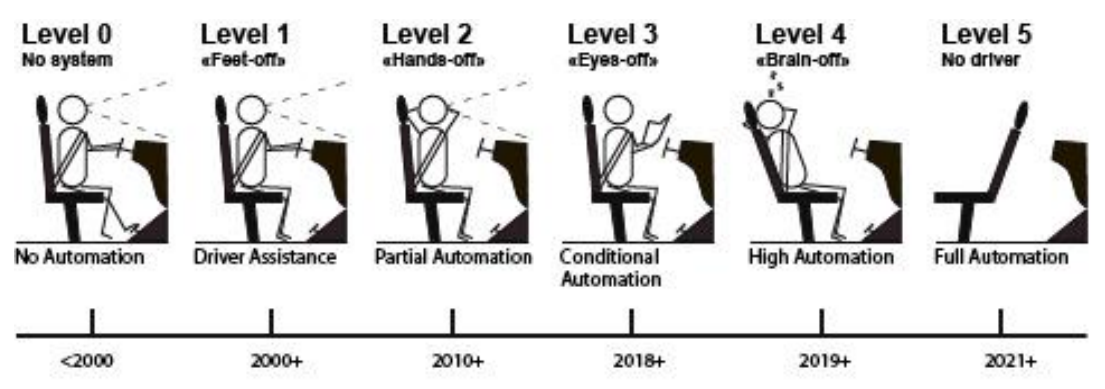

Fig. 1. Automation levels 
The implementation of the autonomous vehicle system includes the following hardware elements: cameras and optical instruments, system-on-chip (SoC), radar, a communication system with other vehicles and infrastructure (V2V/V2I), active remote sensing system LiDAR (Light Detection and Ranging), converters of digital signal to mechanical actions, ultrasonic sensors, GPS, odometric sensors, etc., as well as software elements such as: high-resolution maps, methods of simultaneous localization and map building (SLAM, simultaneous localization and mapping), algorithms for data analysis and processing, recognition of obstacles and objects, prediction of trajectories and actions of moving objects, decision-making algorithms, such as planning routes, making maneuvers, accelerating, braking, taxiing, an operating system for driving a car, a supervisory control system for identifying and eliminating errors, as well as a system for interacting with an external data collection center and cloud services [9].

The development of robotics, machine vision, artificial intelligence, increasing the speed of data processing made it possible to implement autonomous vehicle. But the new concept of transport systems without the active participation of the person who delegated the right to drive to autonomous systems, together with solving a number of traditional issues, brings an even greater number of new problems. These include problems of liability in the case of damage, loss of the ability to drive a vehicle independently, reliability and vulnerability of software, loss of privacy, the danger of using autonomous cars for terrorist activities, the ethical issue of minimizing casualties when making decisions in critical situations etc. [10].

Another important aspect of driving in extreme situations is the study of the mechanical characteristics of the adhesion of the wheels of a moving car to the roadway. The fact is that when performing a speed maneuver while decreasing the distance between the cars, the driver makes decisions based on his experience, may not take into account all conditions, such as abrasion of treads, defects in the road surface, various degrees of moisture in the roadway, etc. This problem is relevant both for those cases when a person is driving a car, and for improving the control algorithms of autonomous vehicles.

\section{Description of the Wheelset Model}

\subsection{Description of the wheelset system without wheel slip}

Consider a wheelset on an inclined plane, shown in Figure 2. This design consists of two homogeneous disks, each with mass $m$ and radius $b$, freely mounted on the axis $\mathrm{O}_{1} \mathrm{O}_{2}$, which is a uniform axle of mass $\mathrm{M}$ and length $2 l$. Thus, disks (wheels) can freely and independently rotate around the axis $\mathrm{O}_{1} \mathrm{O}_{2}$ without friction. 


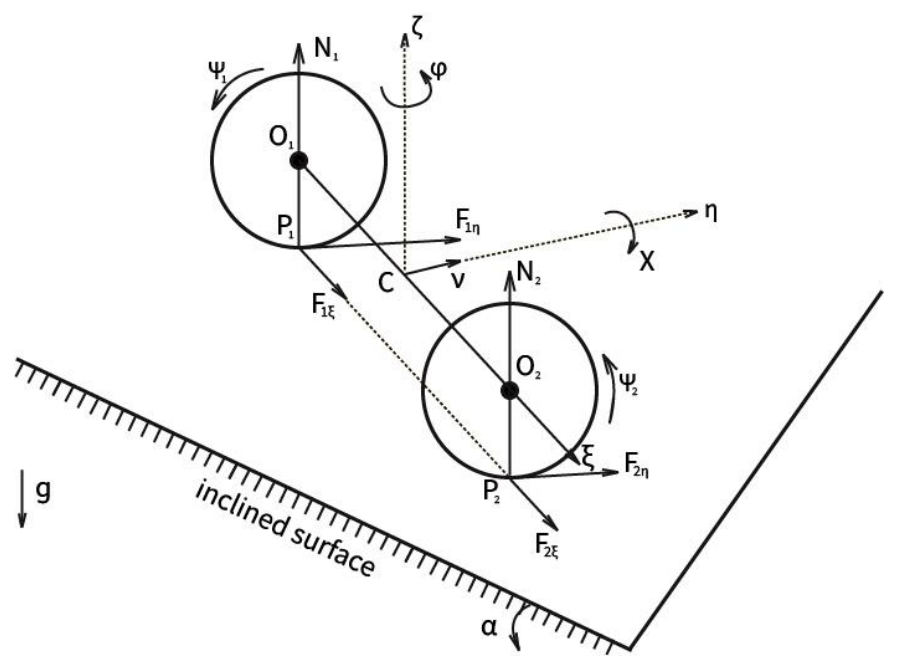

Fig. 2. Wheelset on surface

The described system moves under the influence of gravity along a rough inclined plane (the angle of inclination to the horizontal is $a$ ) so that at the points of contact of the wheels with the plane (points $P_{1}, P_{2}, \mathrm{P} 1, \mathrm{P} 2$, see Fig. 2), and no wheel slip (neither lateral nor longitudinal) occurs.

\subsection{Basic kinematic variables}

Let $\mathrm{C}$ - be the center of mass of the system (it is clear that it is in the middle of the segment $\mathrm{O}_{1} \mathrm{O}_{2}$ ), and $\mathrm{C} \xi \eta \zeta$ - is the coordinate system rigidly connected with the axis $\mathrm{O}_{1} \mathrm{O}_{2}$, moreover $\mathrm{C} \xi$ is directed along the axis $\mathrm{O}_{1} \mathrm{O}_{2}, \mathrm{C} \zeta$ - directed upward along the normally inclined plane, and $\mathrm{C} \eta-$ is perpendicular to $\mathrm{C} \xi$ and $\mathrm{C} \eta$ and forms the right coordinate system with them.

The movement of the wheelset occurs under the influence of gravity and reaction forces of the plane that occur at the contact points $\mathrm{P}_{1}$ and $\mathrm{P}_{2}$. These forces are shown in fig. 2: $\mathrm{N}_{1}, \mathrm{~N}_{2}$ - normal reaction forces, and the values of their projections onto the axis $\mathrm{C} \xi$ should be non-negative $\left(\mathrm{N}_{1} \geqslant \geqslant, \mathrm{~N}_{2 \zeta} \geqslant 0\right), \mathrm{F}_{1 \xi}, \mathrm{F}_{2 \xi}$ - lateral, and $\mathrm{F}_{1 \eta}, \mathrm{F}_{2 \eta}$ - longitudinal forces adhesion (static friction forces). In the future, we will assume that separation does not occur and $F_{1 \eta}, N_{2 \zeta}$ are substantially positive values. In addition, let the friction coefficient of the reference plane be so large that there is no wheel slip at points $\mathrm{P}_{1}, \mathrm{P}_{2}$, thus $\mathrm{V}_{P 1}=\mathrm{V}_{P 2}=0$.

Let $\psi_{1}, \psi_{2}$ be the proper angles of rotation of the wheels relative to the axis $\mathrm{O}_{1} \mathrm{O}_{2}, \varphi$ is the angle of rotation of the axis $\mathrm{O}_{1} \mathrm{O}_{2}$ in an inclined plane, counted counterclockwise from the line of the largest slope (if viewed from the positive end of the axis $\mathrm{C} \xi$ ).

Thus, the angular velocity of the axis $\mathrm{O}_{1} \mathrm{O}_{2}$ (and hence the of system $\mathrm{C} \xi \eta \zeta$ ) in the projections on the axis $\xi, \eta, \zeta$ is:

$$
w_{0}=(0,0, j)
$$


The angular velocity of each of the wheels, in projections on the same axis:

$$
w j=\left(\dot{y}_{j}, 0, j\right), j=1.2
$$

Let's find the speed of point O1, considering it to belong to the wheel 1:

$$
\mathrm{n}_{O_{1}}=\mathrm{n}_{P_{1}}+\left[\mathrm{w}_{1} \times P_{1} O_{1}\right]
$$

Hence, since $n_{P_{1}}=0$, we obtain:

$$
\mathrm{u}_{O_{1} \mathrm{x}}=0, \mathrm{u}_{O_{1} \mathrm{~h}}=-b \dot{y}_{1}, \quad \mathrm{u}_{O_{1} \mathrm{z}}=0
$$

On the other hand, since the point $\mathrm{O} 1$ belongs to the axis $\mathrm{O} 1 \mathrm{O} 2$, we obtain:

$$
\mathrm{n}_{O_{1}}=\mathrm{n}_{C}+\left[\mathrm{w}_{1} \times C O_{1}\right]
$$

or, in projections on the axis $\xi, \eta, \zeta$ we obtain:

$$
\mathrm{u}_{O_{1} \mathrm{x}}=\mathrm{u}_{C \mathrm{x}}, \quad \mathrm{u}_{O_{1} \mathrm{~h}}=\mathrm{u}_{C \mathrm{~h}}-l \mathrm{j}, \quad \mathrm{u}_{O_{1} \mathrm{z}}=\mathrm{u}_{C \mathrm{z}}
$$

From (1.3), (1.4) it follows

$$
\mathrm{u}_{C \mathrm{x}}=\mathrm{u}_{C \mathrm{z}}=0, \quad \mathrm{u}_{C \mathrm{~h}}-l \mathrm{j}=-b \dot{\mathrm{y}}_{1}
$$

Similar logic for the point $\mathrm{O}_{2}$ leads to the relations:

$$
\mathrm{u}_{C \mathrm{x}}=\mathrm{u}_{C \mathrm{z}}=0, \quad \mathrm{u}_{C \mathrm{~h}}-l \mathrm{j}=-b \dot{\mathrm{y}}_{2}
$$

Thus, no wheel slip condition leads to the following conclusions:

1. The speed of point $\mathrm{C}$ has a nonzero projection only onto the longitudinal axis $\mathrm{Ch}$, we will denote it hereafter by $u$, which is

$$
\mathrm{u}_{C \mathrm{x}}=0, \mathrm{u}_{C \mathrm{~h}}=\mathrm{u}, \mathrm{u}_{C \mathrm{z}}=0
$$

2. Two relations are satisfied:

$$
\left\{\begin{array}{l}
\mathrm{u}+l \mathrm{j}=-b \dot{y}_{2} \\
\mathrm{u}-\mathrm{lj}=-b \dot{y}_{1}
\end{array}\right.
$$

\subsection{The equation of motion of wheelset without wheel slip}

The equations of motion of the center of mass of the system in the axes $C x h z$, taking into account (1.1) and (1.4), we obtain:

$$
\left\{\begin{array}{c}
m_{0}(-v \mathrm{j})=F_{1} \mathrm{x}+F_{2} \mathrm{x}+m_{0} g \sin \alpha \sin \mathrm{j} \\
m_{0} \dot{v}=F_{1} \mathrm{~h}+F_{2} \mathrm{~h}+m_{0} g \sin \alpha \cos \mathrm{j}, \\
0=N_{1}+N_{2}-m_{0} g \cos \alpha
\end{array}\right.
$$

Where $m_{0}=M+2 m$ is the aggregate mass of the system. 
Let's denote $J_{\xi}, J_{\mathrm{z}}$ be the axial and equatorial moments of inertia of the wheel $\left(J_{\xi}=\right.$ $\left.m b^{2} / 2, J_{\mathrm{z}}=m b^{2} / 4\right)$, respectively, $J_{1}$ is the mass moment of inertia of the axle relative to axis $C \mathrm{z}$ (or $C \mathrm{~h})\left(J_{1}=M l^{2} / 3\right)$. Using the symmetry of the system and formulas (1.1) and (1.2) for the angular velocities, we obtain that the kinetic moment is follows:

$$
K_{C \xi}=J_{\xi}\left(\dot{\mathrm{y}}_{1}+\dot{\mathrm{y}}_{2}\right), K_{C \mathrm{~h}}=0, K_{C \mathrm{z}}=J_{0} \ddot{\mathrm{j}},
$$

Where $J_{0}=J_{1}+2 J_{\mathrm{z}}+2 m l^{2}$. Using (1.8) and relation (1.1) for the angular velocity $C \mathrm{xhz}$, we obtain the equations of the theorem on the change in the kinetic momentum $K_{C}$ in the $C$ xhz axes:

$$
\left\{\begin{array}{c}
J_{\xi}\left(\ddot{\mathrm{y}}_{1}+\ddot{\mathrm{y}}_{2}\right)=b\left(F_{1 \mathrm{~h}}+F_{2 \mathrm{~h}}\right), \\
J_{\xi}\left(\dot{\mathrm{y}}_{1}+\dot{\mathrm{y}}_{2}\right)=\left(N_{1}-N_{2}\right) l-b\left(F_{1 \xi}+F_{2 \xi}\right), \\
J_{0} \ddot{\mathrm{j}}=l\left(F_{2 \mathrm{~h}}-F_{1 \mathrm{~h}}\right) .
\end{array}\right.
$$

Now, we write down the equations for changing the kinetic moment for each of the wheels separately with respect to the $\xi$ axis, under the assumption that they rotate freely on the $\xi$ axis and there are no rotations around $\mathrm{Ch}$ axis, i.e.:

$$
J_{\xi} \ddot{y}_{j}=b F_{j \mathrm{~h}}, j=1,2 .
$$

Thus, we obtain a closed system of 10 equations (1.6)-(1.10) for determining 10 variables: $v, j, y_{1}, y_{2}, N_{1}, N_{2}, F_{1 \xi}, F_{2 \xi}, F_{1 \mathrm{~h}}, F_{2 \mathrm{~h}}$.

\subsection{Analysis of the system of equations (1.6)-(1.10)}

Let the initial conditions be:

$$
\left\{v_{C}(0), \mathrm{j}(0), \mathrm{j}(0), \dot{\mathrm{y}}_{1}(0), \dot{\mathrm{y}}_{2}(0)\right\}
$$

such that equalities (1.5) and (1.6) are satisfied at $\mathrm{t}=0$ (the moment of start of the wheelset movement in the plane, so that the kinematic coupling conditions are not violated). It is required to determine the solution of system (1.6)-(1.10), which satisfies the continuity condition: $N_{j}>0, j=1,2$. In a more accurate statement, if the friction coefficient $f$ of the plane with wheels is specified, it is also necessary to satisfy Coulomb's inequalities in the process of motion:

$$
F_{j \xi}^{2}+F_{j \mathrm{~h}}^{2} \leq f^{2} N_{j}^{2}, j=1,2
$$

This kind of problem was considered for the Chapylygin sleigh on an inclined plane with friction in work [11].

As shown in [12], the solution has the form:

$$
v(t)=v_{0}+\frac{g \sin a}{\omega_{0}\left(1+\frac{2 p^{2}}{b^{2}}\right)}\left[\sin \left(\omega_{0} t+\varphi_{0}\right)-\sin \varphi_{0}\right]
$$

Let $\mathrm{x}$ be the coordinate of the center of mass $\mathrm{C}$ along the line of the largest slope (down the inclined plane), $\mathrm{y}$ - be the coordinate perpendicular to it, then we have: 


$$
\dot{x}=v \cos \varphi, \dot{y}=v \sin \varphi .
$$

Integration of the last equalities leads to the conclusion that the center of mass of motion along the cycloid perpendicular to the line of the largest slope, that is, the wheelset does not roll down.

\subsection{Critical situations}

Example:

$$
\frac{\omega_{0} v_{0}}{g}\left(1+\frac{2 p^{2}}{b^{2}}\right)<\frac{1}{b} \cos a-2 \sin a+\sin a \sin \varphi_{0} .
$$

For example, for $v_{0}=0, \varphi_{0}=0$ we have $\operatorname{tga}<l /(2 b)$, that is, a rollover can happen for sufficiently large $a$ : $\operatorname{tg} a>l /(2 b)$.

If inequality (1.15) is violated, then there will definitely come a point in time that a normal reaction is zero, for example, $N_{1}$. So, let $N_{1}\left(t_{1}\right)=0$ and further movement is possible only with separation of wheel 1 (while maintaining the contact of wheel 2). Consider the beginning of such a separation, that is, the resulting rotation of the wheelset around the axis $C$ h. We will determine such a rotation by the angle $x$, count it counterclockwise, if we look from the positive part of the axis $C \mathrm{~h}$ (see Fig. 2). Considering the further movement, we assume that $x \approx 0, \dot{x} \approx 0, \ddot{x} \neq 0$. Then we have:

$$
\begin{gathered}
v_{C J \xi}=0, v_{C h}=v, v_{C z}=l \dot{x} ; \\
K_{C J \xi}=J_{\xi}\left(\dot{\mathrm{y}}_{1}+\dot{\mathrm{y}}_{2}\right), K_{C \mathrm{~h}}=J_{0} \dot{x}, K_{C \mathrm{z}}=J_{0}, \\
\omega_{0}=(0, \dot{x}, \dot{\varphi}) .
\end{gathered}
$$

After several operations we obtain:

$$
\begin{gathered}
\left(\frac{J_{0}+m_{0} l^{2}}{m_{0}}\right) \dddot{x}=\dot{v} \dot{\varphi}\left(b+\frac{2 p^{2}}{b}\right)+v \ddot{\varphi}\left(b+\frac{2 p^{2}}{b}\right)+g b \operatorname{sinacos} \varphi \dot{\varphi}= \\
=g b \operatorname{sinacos} \varphi\left[\left(1+\frac{2 p^{2}}{b}\right)\left(\dot{\varphi} \frac{p_{0}^{2} b^{2}+p^{2} l^{2}}{p^{2} p_{0}^{2}+p_{0}^{2} b^{2}+p^{2} l^{2}}-v \frac{p^{2} l}{p^{2} p_{0}^{2}+p_{0}^{2} b^{2}+p^{2} l^{2}}\right)+\dot{\varphi}\right] \\
= \\
=\text { gbsinacos } \varphi\left[\left(1+\frac{2 p^{2}}{b}\right) \frac{\dot{\varphi}\left(p_{0}^{2} b^{2}+p^{2} l^{2}\right)-v p^{2} l}{p^{2} p_{0}^{2}+p_{0}^{2} b^{2}+p^{2} l^{2}}+\dot{\varphi}\right], \\
\text { where } p^{2}=\frac{J_{\xi}}{m_{0}}, p_{0}^{2}=\frac{J_{0}}{m_{0}} .
\end{gathered}
$$

It is clear that choosing $\omega_{0}$ small enough, we can achieve large $v\left(t_{1}\right)$, and formula (1.13) then gives us $\dddot{x}<0$, that is, a paradoxical situation arises when the separation occurs. These considerations lead us to the conclusion that, in cases where there can be a zeroing of the normal reaction, it is necessary to take into account the finite coefficient 
of friction and consider the process of movement of the wheelset with possible transitions to the situation of wheel slippage, which, of course, complicates the solution of the problem.

\section{Description of the Carriage Model}

\subsection{Model description}

Figure 3 shows a flat wheel model of two wheelsets $A_{1} A_{2}, B_{1} B_{2}$, connected at their centers of mass by a rigid axle $A B$. The joints in connection points $A$ and $B$ are assumed to be ideal and cylindrical, and the rolling of wheels $A_{1}, A_{2}, B_{1}, B_{2}$ along the supporting plane occurs without slipping, i.e. a nonholonomic connection is imposed.

In this model, we assume (same as in [1-4]), that this coupling is realized only by the transverse forces $F_{A}$ and $F_{B}$, applied to $A_{1} A_{2}$ and $B_{1} B_{2}$, respectively (see Fig. 3). The consideration of longitudinal forces was considered, for example, in paper [7].

The presence of a non-holonomic connection gives the right to assume that the velocities of the centers of mass of the wheelsets, denoted as $v_{A}$ and $v_{B}$, are always directed perpendicular to the corresponding axes, i.e. $v_{A} \perp A_{1} A_{2}, v_{B} \perp B_{1} B_{2}$ (see Fig.3).

\subsection{Description of symbols}

Further, the following notation is introduced: $\theta_{1}, \theta_{2}-$ the deflection angles of the wheelsets $A_{1}, A_{2}, B_{1}, B_{2}$ from the longitudinal axis $\xi$ of the flat wheel model, and $\theta_{1}$ is counted clockwise, and, $\theta_{2}$ is counterclockwise; $A C=a, C B=b, C$ is the center of mass of the entire system, which we assume to be unchanged, that $a=$ const, $b=$ const; $\varphi$ is the angle of rotation of the axle $\mathrm{AB}$ relative to the invariably oriented axis $C_{x}$, counted counterclockwise; $\mathrm{P}$ is the instantaneous velocity center of the axle $\mathrm{AB}$, which, due to nonholonomic constraints, is the intersection of lines $A_{1} A_{2}$ and $B_{1} B_{2}$; $m_{A B}, m_{A}, m_{B}$ are the masses of axle $\mathrm{AB}$, of wheelsets $A_{1}, A_{2}, B_{1}, B_{2} ; J_{A B}$ is the moment of inertia of the axle $\mathrm{AB}$ together with the concentrated masses $m_{A}$ and $m_{B}$ at its ends relative to point $\mathrm{C} ; J_{A}, J_{B}$ are the moments of inertia of the wheel pairs, respectively $A_{1}, A_{2}, B_{1}, B_{2}$ relative to the own centers of mass $\mathrm{A}$ and $\mathrm{B} ; C_{\xi \mathrm{h}}$ is the coordinate system rigidly connected with the axle $\mathrm{AB}$. 


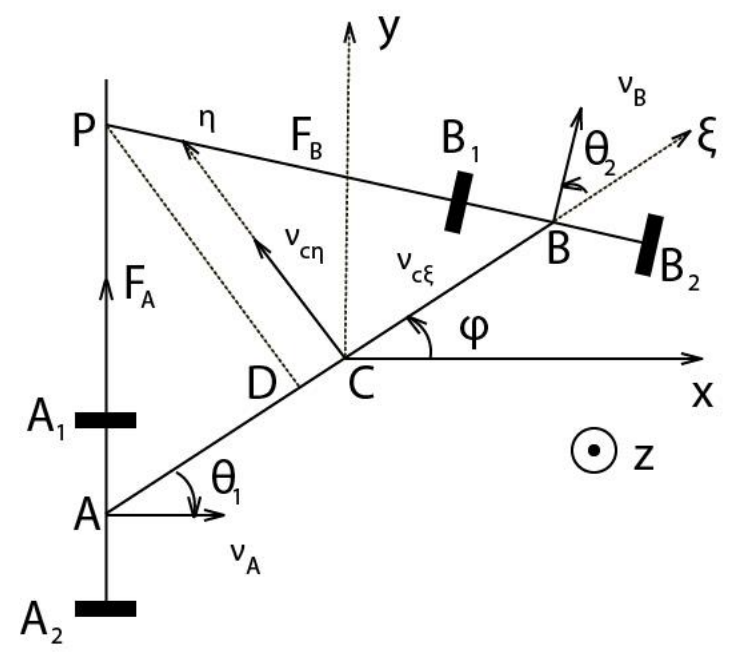

Fig. 3. Carriage on surface

\subsection{Equations of motion}

For the three angles $\varphi, \theta_{1}, \theta_{2}$ we can write three equations of dynamics that do not contain unknown forces and reaction forces in the hinges $\mathrm{A}$ and $\mathrm{B}$ :

$$
K_{P Z}+m\left[v_{p} \times v_{c}\right]_{z}=0,
$$

$$
\begin{aligned}
& J_{A}\left(\ddot{\varphi}-\ddot{\theta}_{1}\right)=M_{A z} \\
& J_{B}\left(\ddot{\varphi}+\ddot{\theta}_{2}\right)=M_{B z}
\end{aligned}
$$

Equation (2.1) is the equation of the kinetic moment relative to the moving point $\mathrm{P}$; and equations (2.2) and (2.3) are the equations of kinetic moments of wheel sets relative to their own centers of mass. The following notation is introduced in these equations: $m=m_{A B}+m_{A}+m_{B}$ is the total mass of the system; $K_{P Z}$ is the kinetic moment of the system relative to the point $\mathrm{P}, v_{p}$ is the speed of the point $\mathrm{P}$ (as geometric relative to the motionless coordinate system), $v_{c}$ is the velocity of the center of mass $\mathrm{C} ; M_{A Z}, M_{B Z}$ control moments in joints $\mathrm{A}$ and $\mathrm{B}$ (if there are any). Let $\mathrm{D}$ be the projection of the point $\mathrm{P}$ onto the axle $\mathrm{AB}$ (see Fig 3). Denote $C D=\xi_{0}$. Then we have, in accordance with Koenig's theorem:

$$
\begin{aligned}
& K_{P Z}=K_{\mathrm{cz}}+m\left(v_{c \xi} \cdot P D+v_{c \mathrm{~h}} \cdot C D\right) \\
& K_{\mathrm{cz}}=J_{A B \dot{\varphi}}+J_{B}\left(\mathrm{j}+\dot{\theta_{2}}\right)+J_{A}\left(\mathrm{j}-\dot{\theta_{1}}\right)
\end{aligned}
$$

After several operations we obtain: 


$$
\begin{gathered}
\dddot{\mathrm{j}}\left\{J_{A B}+\frac{m}{\left(\operatorname{tg} \theta_{1}+\operatorname{tg} \theta_{2}\right)^{2}}\left[(a+b)^{2}+\left(a \operatorname{tg} \theta_{2}-b \operatorname{tg} \theta_{1}\right)^{2}\right]\right\}- \\
-\frac{m \dot{\varphi}(a+b)}{\left(\operatorname{tg} \theta_{1}+\operatorname{tg} \theta_{2}\right)^{3}}\left[\frac{\theta_{2}}{\cos ^{2} \theta_{2}}\left(a+b-a \operatorname{tg} \theta_{1} \operatorname{tg} \theta_{2}+b \operatorname{tg} \theta_{1} \theta_{1}\right)\right. \\
\left.+\frac{\theta_{1}}{\cos ^{2} \theta_{1}}\left(a+b+a \operatorname{tg}^{2} \theta_{2}-b \operatorname{tg} \theta_{1} \operatorname{tg} \theta_{2}\right)\right]=-M_{A Z}-M_{B Z} .
\end{gathered}
$$

Equations (2.2) - (2.4) form a closed (with respect to $\varphi, \theta_{1}, \theta_{2}$ ) system of equations.

\subsection{Case of the equation with fixed angles}

We control the movement of the wheelset, that $M_{A z}$ and $M_{B Z}$ so that $\theta_{1}=\theta_{2}=0$ (the same deviations of the wheelsets, but in different directions). Then from (2.2), (2.3) we get:

$$
M_{A z}+M_{B z}=\left(J_{A}+J_{B}\right) \ddot{\varphi}
$$

And from equation (2.4) we get:

$$
\begin{aligned}
& \ddot{j}\left\{J_{A B}+J_{A}+J_{B}+\frac{m}{4 \operatorname{tg}^{2} \theta}\left[(a+b)^{2}+\operatorname{tg}^{2} \theta(a-b)^{2}\right]\right\}- \\
& -\frac{m \dot{\varphi}(a+b)}{4 t g^{3} \theta}\left[\frac{\theta}{\cos ^{2} \theta}(a+b)\right]=0 .
\end{aligned}
$$

The energy integral takes the form of:

$2 T=\mathrm{j}^{2}\left[J_{A B}+\frac{(a-b)^{2}+\left(a \operatorname{tg} \theta_{2}+b t g \theta_{1}\right)^{2}}{\left(\operatorname{tg} \theta_{1}+\operatorname{tg} \theta_{2}\right)^{2}}\right]=h_{0}^{2}=$ const.

\subsection{Fixed wheelset}

Consider a special case: a fixed wheelset $A_{1}, A_{2}$, that $\theta_{1}=0$, then the energy integral (2.6) has the form of:

$$
\begin{gathered}
\mathrm{j}^{2}\left[J_{A B}+m \frac{(a+b)^{2}+a^{2} \operatorname{tg}^{2} \theta_{2}}{\operatorname{tg}^{2} \theta_{2}}\right]=h_{0}^{2}=> \\
\mathrm{j}^{2}\left[\left(J_{A B}+m a^{2}\right)+m(a+b)^{2} c t g^{2} \theta_{2}\right]=h_{0}^{2} .
\end{gathered}
$$

Equation (2.3) with $M_{B Z}=0$ gives us:

$$
\dot{\varphi}=\omega_{0}-\dot{\theta}_{2}
$$

and from (2.7) we obtain $A^{2}=J_{A B}+m a^{2}, B^{2}=m(a+b)^{2}, \lambda=\frac{h_{0}}{\omega_{0}}, x=\operatorname{tg} \theta_{2}$ : 


$$
\begin{aligned}
& \frac{d \theta_{2}}{d t}=\frac{\omega_{0} \sqrt{B^{2}+A^{2}+\operatorname{tg}^{2} \theta_{2}}-h_{0} t g \theta_{2}}{\sqrt{B^{2}+A^{2} \operatorname{tg}^{2} \theta_{2}}} \rightarrow \\
& I=\int \frac{d \theta_{2} \sqrt{B^{2}+A^{2} t g^{2} \theta_{2}}}{\sqrt{B^{2}+A^{2} \operatorname{tg}^{2} \theta_{2}}-\lambda \operatorname{tg} \theta_{2}}=\omega_{0} t+C
\end{aligned}
$$

By denoting $x=\operatorname{tg} \theta_{2}$, we obtain:

$$
I=\int \frac{d x}{1+x^{2}}+\lambda \int \frac{x d x \sqrt{B^{2}+A^{2} \operatorname{tg}^{2} \theta_{2}}}{\left(1+x^{2}\right)\left[B^{2}+\left(A^{2}-\lambda^{2}\right) x^{2}\right]}+\lambda^{2} \int \frac{x^{2} d x}{\left(1+x^{2}\right)\left[B^{2}+\left(A^{2}-\lambda^{2}\right) x^{2}\right]} .
$$

It is easy to show that all these integrals are taken in elementary functions. Thus, in this case, the equations of motion of the system are integrated in time functions for any parameter values and initial conditions.

\subsection{Controllable wheelset}

$$
\begin{gathered}
\dot{\varphi}\left\{J_{A B}+J_{A}+J_{B}+\frac{m}{4}\left[\frac{(\dot{a}+b)^{2}}{\operatorname{tg}^{2} \theta}+\operatorname{tg}^{2} \theta(a-b)^{2}\right]\right\}-\frac{m \dot{\varphi}(a-b)^{2}}{4 \operatorname{tg}^{2} \theta} \cdot \frac{\dot{\theta}}{\cos ^{2} \theta}=0 ; \\
\ddot{\varphi}=\frac{1}{2} \frac{d\left(\dot{\varphi}^{2}\right)}{d \varphi} ; \dot{\varphi} \dot{\theta}=(\dot{\varphi})^{2} \frac{d \theta}{d \varphi} ; A=\frac{m}{4}(a-b)^{2}+J_{A B}+J_{A}+J_{B} ; \\
d\left(\dot{\varphi}^{2}\right)\left[A+\frac{m(a+b)^{2} \operatorname{ctg}^{2} \theta}{4}\right]-\frac{m(a+b)^{2}}{2 \operatorname{tg}^{3} \theta \cos ^{2} \theta} \cdot(\dot{\varphi})^{2} d \theta=0 \rightarrow \\
\frac{d\left(\dot{\varphi}^{2}\right)}{\dot{\varphi}^{2}}=d \theta \cdot \frac{m(a+b)^{2}}{2 \operatorname{tg}^{3} \theta \cos ^{2} \theta\left[A+\frac{m(a+b)^{2} c t g^{2} \theta}{4}\right]} \\
\dot{\varphi}=\frac{C_{0} \operatorname{tg} \theta}{\sqrt{\operatorname{Atg}^{2} \theta+B}} .
\end{gathered}
$$

The resulting equation is the main equation for the movement of a controlled wheelset.

\section{Conclusion}

In this paper we've found equation for the movement of a wheelset without wheel slip, where two wheels freely mounted on an axis and moving along an inclined plane in the field of gravity, another equation of movement of a wheel model, which, under certain assumptions, can be a four-wheeled carriage. In addition to dynamics of continuous motion without wheel slip, critical situations were observed associated with wheel 
slip and separation of the carriage wheels from the plane. The related parameters were found and considered.

\section{Acknowledgement}

The work has been supported by Russian Foundation for Basis Research, Grant No. 17-29-03419.

\section{$6 \quad$ References}

[1] Buslaev A P, Yashina M V. Modern information-communication methods of analysis and models of complex socio-technical systems. InPaper presented at the IMCIC 2018-9th International Multi-Conference on Complexity, Informatics and Cybernetics, Proceedings 2018 (Vol. 2, pp. 77-82).

[2] Lukanin V N, Buslaev A P, Novikov A V, Yashina M V. Traffic flows modelling and the evaluation of energy-ecological parameters. Part I. International journal of vehicle design. 2003 Jan 1;33(4):381-99. https://doi.org/10.1504/ijvd.2003.003578

[3] Mawson A R. Mass panic and social attachment: The dynamics of human behavior. Routledge, 2017 \& Baker GW Schultz: PANIC BEHAVIOR: DISCUSSION AND READINGS (Book Review) // Social Forces. - 1965. - T. 44. - No. 1. - P. 143. https://doi.org/10.432 4/9781351153201

[4] Schultz D P. Panic behavior: discussion and readings. New York, Random House; 1964.

[5] Shuvalov I., Russian Government Website on Dangerous Driving, 2016. (Available at http://xn--80aegccaes3bfdcci5a.xn--p1ai/).

[6] Tarasov A Iu., Mironov V L. On the Question of the Definition of "Dangerous Driving" Actual Problems of the Administrative Policing. Krasnodar, Krasnodar University of the Ministry of the Interior of the Russian Federation Publ., 2015, pp. 142-147.

[7] Tishkin V F, Yashina M V, Moseva M S, Yashin V B. Method of the GPS tracking analysis for extraction of geometrical properties. In2018 IEEE International Conference" Quality management, transport and information security, information technologies"(IT\&QM\&IS) 2018 Sep 24, pp. 266-270. IEEE. https://doi.org/10.1109/itmqis.2018.8524946

[8] Autonomous Driving | Moonshot Project with Quantum Leap from Hardware to Software \& AI Focus 2019 Deloitte (available at https://www2.deloitte.com/content/dam/Deloitte/be/ Documents/Deloitte_Autonomous-Driving.pdf ) https://doi.org/10.1093/ww/97801995408 $\underline{84.013 .45187}$

[9] Shi W, Alawieh M B, Li X, Yu H. Algorithm and hardware implementation for visual perception system in autonomous vehicle: A survey. Integration. 2017 Sep 1;59:148-56. https://doi.org/10.1016/j.vlsi.2017.07.007

[10] Bonnefon J F, Shariff A, Rahwan I. The social dilemma of autonomous vehicles. Science. 2016 Jun 24;352(6293):1573-6. https://doi.org/10.1126/science.aaf2654

[11] Zhuravlev V. F., Fuvaev N. A. The mechanics of systems with non-restraining connections. Editor-in-chief of the Academy of RAS Klimov DM. 1993.

[12] Rosenblat G. M. To the dynamics of non-holonomic models of wheeled carriages. Bulletin of the Udmurt University. Maths. Mechanics. Computer science. 2008 (3): 99-108. 
Paper-Model of Carriage Movement on Plane with Dry Friction Forces

\section{Authors}

G.M. Rosenblat is from Moscow Automobile and Road Construction State Technical University (MADI), Moscow, Russia

V.F. Tishkin is a Professor and Deputy Director at Keldysh Institute of Applied Mathematics, Moscow, Russia.

V.B. Yashin is from Moscow Technical University of Communications and Informatics (MTUCI), Moscow, Russia

Article submitted 2020-04-03. Resubmitted 2020-05-17. Final acceptance 2020-05-18. Final version published as submitted by the authors. 